\title{
Analysis of the efficacy of lenalidomide in patients with intermediate-1 risk myelodysplastic syndrome without 5q deletion
}

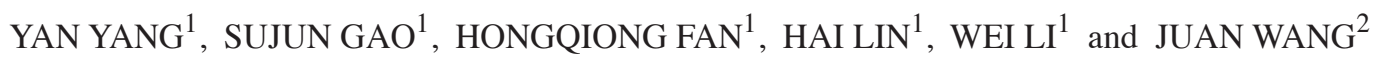 \\ ${ }^{1}$ Department of Haematology and Oncology, The First Hospital of Jilin University, Changchun, Jilin 130021; \\ ${ }^{2}$ Department of Oncology, People's Hospital of Jilin Province, Changchun, Jilin 130021, P.R. China
}

Received March 17, 2013; Accepted June 18, 2013

DOI: $10.3892 /$ etm.2013.1218

\begin{abstract}
The aim of this study was to evaluate the efficacy and adverse effects of lenalidomide in the treatment of intermediate-1 risk non-5q deletion [non-del (5q)] myelodysplastic syndrome (MDS). A total of 30 patients with MDS were classified through G-banding chromosome karyotype analysis and fluorescence in situ hybridization (FISH). According to the International Prognostic Scoring System scores, among the 30 patients, 23 and seven cases had scores of 0.5 and 1.0 , respectively. Lenalidomide $\left(\operatorname{Revlimid}^{\circledR)}, 10 \mathrm{mg} /\right.$ day) was administered for 21 days every 28 days. All 30 cases were treated with lenalidomide for at least three cycles, including 20 cases with four cycles. The patients did not require erythropoietin, cyclosporine or iron chelation treatments. Statistical analysis was performed using SPSS statistical software version 13.0, and comparisons among groups were conducted using a t-test. The efficacy of lenalidomide was demonstrated in patients with intermediate-1 risk non-del (5q) MDS. Peripheral blood cell counts were improved following treatment, and absolute neutrophil, haemoglobin and platelet counts increased following 2-4 cycles of treatment. All patients became stable having undergone three cycles of treatment; however, 17 patients with chromosomal abnormalities had no cytogenetic response to the treatment, as confirmed through the FISH test. Patients with intermediate-1 risk non-del (5q) MDS treated with lenalidomide did not achieve complete haematological remission, although they demonstrated haematological improvement.
\end{abstract}

Correspondence to: Dr Wei Li, Department of Haematology and Oncology, The First Hospital of Jilin University, 71 Xinmin Street, Changchun, Jilin 130021, P.R. China

E-mail: cnliwei@yeah.net

Dr Juan Wang, Department of Oncology, People's Hospital of Jilin Province, 1183 Gongnong Street, Changchun, Jilin 130021, P.R. China

E-mail: jcnwang@yeah.net

Key words: lenalidomide, myelodysplastic syndrome

\section{Introduction}

Myelodysplastic syndrome (MDS) is a heterogeneous clonal haematopoietic stem cell disorder characterised by ineffective haematopoiesis and a high risk of progression to acute myeloid leukemia (AML). The ineffective haematopoiesis results in one or more peripheral cytopaenias, dysplasic changes in the haematopoietic cell lines and typical cytogenetic abnormalities (1). Conventional drugs, including haematopoietic cytokines and chemotherapy drugs, have shown little efficacy in the treatment of the disease, although in recent years, numerous new drugs have been listed or have been subject to clinical trials. Three drugs have recently been approved by the US Food and Drug Administration (FDA) for the treatment of MDS within the last seven years: lenalidomide, azacytidine and decitabine. Lenalidomide is predominantly used for the treatment of patients with MDS with $5 q$ deletion [del (5q)] cytogenetic abnormalities. However, the 2009 version of the National Comprehensive Cancer Network (NCCN) guidelines also recommended lenalidomide for the management of low/intermediate-1 risk MDS.

A number of studies have demonstrated that lenalidomide treatment is effective in patients with MDS with del (5q), and may increase the number of peripheral blood cells, thus enabling patients to undergo a reduced number of infusions, and improving the quality of life (2-4). However, there have been few investigations into the efficacy of lenalidomide for patients with intermediate-1 risk MDS without del $(5 q)$, particularly amongst Chinese individuals. Since March 2009, the treatment of patients with low/intermediate-1 risk MDS at the Department of Haematology and Oncology of The First Hospital of Jilin University (Changchun, China) has included the administration of lenalidomide. By March 2011, 30 patients had received this treatment. The clinical data obtained from the patients are summarised in the current study.

\section{Patients and methods}

Patients. A total of 30 patients in the Department of Haematology and Oncology of The First Hospital of Jilin University were diagnosed with MDS through peripheral blood counts, bone marrow smears, bone marrow biopsies 
Table I. Clinical data of 30 patients with MDS

\begin{tabular}{|c|c|c|c|c|c|c|c|}
\hline Case no. & $\begin{array}{c}\text { Gender } \\
(\mathrm{M} / \mathrm{F})\end{array}$ & $\begin{array}{c}\text { Age } \\
\text { (years) }\end{array}$ & $\begin{array}{l}\text { Time from diagnosis to } \\
\text { treatment (months) }\end{array}$ & WHO classification & Chromosome karyotype & $\begin{array}{l}\text { FISH } \\
\text { result }\end{array}$ & $\begin{array}{l}\text { IPSS } \\
\text { score }\end{array}$ \\
\hline 1 & $\mathrm{~F}$ & 13 & 0 & MDS-U & $47 \mathrm{XX},+8[9] / 46 \mathrm{XX}[11]$ & +8 & 1.0 \\
\hline 2 & M & 31 & 4 & RA & $46, X Y[20]$ & $12 \mathrm{p}^{-}$ & 0.5 \\
\hline 3 & M & 35 & 2 & RA & $46, X Y[20]$ & $12 \mathrm{p}^{-}$ & 0.5 \\
\hline 4 & $\mathrm{~F}$ & 37 & 2 & RCMD & $46, \mathrm{XX}[20]$ & Negative & 0.5 \\
\hline 5 & $\mathrm{~F}$ & 40 & 4 & RCMD & $46, X X, \operatorname{del}(20)\left(q^{11}\right)[6] / 46, X X[14]$ & $20 \mathrm{q}^{-}$ & 0.5 \\
\hline 6 & $\mathrm{~F}$ & 42 & 3 & RCMD & $46, X X, \operatorname{del}(20)\left(q^{11}\right)[5] / 46, X X[15]$ & $20 \mathrm{q}^{-}$ & 0.5 \\
\hline 7 & M & 45 & 6 & RCMD & Failure & +8 & 1.0 \\
\hline 8 & $\mathrm{~F}$ & 46 & 3 & RCMD & $46, X X, \operatorname{del}(20)\left(q^{13}\right)[7] / 46, X X[13]$ & $20 \mathrm{q}^{-}$ & 0.5 \\
\hline 9 & M & 50 & 3 & RCMD & $46, \mathrm{XX}[20]$ & Negative & 0.5 \\
\hline 10 & M & 51 & 2 & RA & $46, X Y, \operatorname{dup}(1)\left(q^{22-31}\right)[8] / 46, X Y[12]$ & Negative & 0.5 \\
\hline 11 & $\mathrm{~F}$ & 51 & 1 & RCMD & $46, \mathrm{XX}[20]$ & Negative & 0.5 \\
\hline 12 & $\mathrm{~F}$ & 51 & 3 & RCMD & $47, \mathrm{XX},+8[7] / 46 \mathrm{XX}[13]$ & +8 & 1.0 \\
\hline 13 & M & 52 & 6 & MDS-U & $46, X Y, \operatorname{del}(20)\left(q^{13}\right)[5] / 46, X Y[15]$ & $20 \mathrm{q}^{-}$ & 0.5 \\
\hline 14 & M & 52 & 7 & RCMD & $46, X Y, \operatorname{del}(20)\left(q^{11}\right)[7] / 46, X Y[13]$ & $20 \mathrm{q}^{-}$ & 0.5 \\
\hline 15 & M & 52 & 8 & RCMD & $46, X Y[20]$ & $12 \mathrm{p}^{-}$ & 1.0 \\
\hline 16 & $\mathrm{~F}$ & 52 & 2 & RCMD & Failure & Negative & 0.5 \\
\hline 17 & $\mathrm{~F}$ & 52 & 5 & RA & $46 \times X[15]$ & Negative & 0.5 \\
\hline 18 & M & 53 & 4 & RCMD & $46, X Y[20]$ & Negative & 0.5 \\
\hline 19 & $\mathrm{~F}$ & 53 & 6 & MDS-U & $46, X X, \operatorname{del}(20)\left(\mathrm{q}^{11}\right)[7] / 46, \mathrm{XY}[13]$ & $20 \mathrm{q}^{-}$ & 0.5 \\
\hline 20 & M & 54 & 7 & RT & $46 X Y[20]$ & $12 \mathrm{p}^{-}$ & 0.5 \\
\hline 21 & $\mathrm{~F}$ & 54 & 8 & RCMD & Failure & +8 & 1.0 \\
\hline 22 & M & 56 & 9 & RCMD & $46, X Y[20]$ & Negative & 0.5 \\
\hline 23 & $\mathrm{~F}$ & 57 & 2 & MDS-U & $46, X X, \operatorname{del}(20)\left(\mathrm{q}^{11}\right)[12] / 46, \mathrm{XY}[8]$ & $20 \mathrm{q}^{-}$ & 0.5 \\
\hline 24 & M & 59 & 2 & RARS & $46, X Y[20]$ & Negative & 0.5 \\
\hline 25 & M & 60 & 1 & RCMD & $46, X Y[20]$ & Negative & 0.5 \\
\hline 26 & $\mathrm{~F}$ & 62 & 3 & MDS-U & $47, \mathrm{XX},+8[5] / 46 \mathrm{XX}[15]$ & +8 & 1.0 \\
\hline 27 & $\mathrm{~F}$ & 65 & 2 & RCMD & $46, \mathrm{XX}[20]$ & Negative & 0.5 \\
\hline 28 & $\mathrm{~F}$ & 66 & 6 & RCMD & Failure & +8 & 1.0 \\
\hline 29 & $\mathrm{~F}$ & 69 & 9 & RA & $46, \mathrm{XX}[20]$ & Negative & 0.5 \\
\hline 30 & M & 83 & 3 & RA & $46, X Y[20]$ & Negative & 0.5 \\
\hline
\end{tabular}

MDS, myelodysplastic syndrome; M, male; F, female; WHO, World Health Organization; FISH, fluorescence in situ hybridization; IPSS, International Prognostic Scoring System; MDS-U, MDS-unclassified; RA, refractory anemia; RCMD, refractory cytopaenia with multilineage dysplasia; RT, refractory thrombocytopaenia; RARS, refractory anemia with ring sideroblasts.

and cytogenetic analyses. This study was conducted in accordance with the Declaration of Helsinki and with approval from the Ethics Committee of the First Hospital of Jilin University. Written informed consent was obtained from all participants. All patients were classified through G-banding chromosome karyotype analysis combined with a fluorescence in situ hybridization (FISH) test $(+8,-5 / 5 q-,-7 / 7 q-, 20 q-,-Y$ and $-12 / 12 \mathrm{p}$ - were selected as the cytogenetic probes in the FISH method). Karyotype anomalies were found in 11 cases, including seven with $20 \mathrm{q}-$, three with +8 , one with dup (1) (q22 31), and four cases that failed in the karyotype analysis. The FISH test revealed anomalies in 17 cases, including six with +8 , seven with $20 \mathrm{q}$-, four with $12 \mathrm{p}$ - and 13 cases with negative results. The patients included 14 males and 16 females, with ages ranging from 13 to 83 years old (median age, 52 years). The duration between diagnosis and treatment ranged from
0 to 9 months (mean, 4.1 months). Six cases of refractory anemia (RA), one case of refractory thrombocytopaenia (RT), one case of refractory anemia with ring sideroblasts (RARS), 17 cases of refractory cytopaenia with multilineage dysplasia (RCMD) and five cases of MDS-unclassified (MDS-U) were identified according to the World Health Organization (WHO) classification system (5). In addition, the International Prognostic Scoring System (IPSS) was used evaluate the 30 patients, and of these, 23 had a score of 0.5 and seven had a score of 1.0 (5). The average erythropoietin (EPO) levels were $>500 \mathrm{mU} / \mathrm{ml}$ in all the patients; in addition, two out of 27 cases tested positive for human leukocyte antigen (HLA)-DR15.

Management. Lenalidomide (Revlimid ${ }^{\circledR}, 10 \mathrm{mg} /$ day) was administered for 21 days every 28 days. All 30 cases were treated with at least three cycles, with 20 cases being treated 
Table II. Changes in peripheral blood cell counts following treatment $(n=30)$.

Following treatment

\begin{tabular}{lccc} 
Peripheral blood cell counts & Prior to treatment & 2 cycles & 4 cycles \\
\hline Absolute neutrophil count $\left(\times 10^{9} / 1\right)$ & $0.95 \pm 0.47$ & $1.02 \pm 0.59$ & $1.28 \pm 0.84$ \\
Haemoglobin $(\mathrm{g} / \mathrm{l})$ & $59.1 \pm 16.1$ & $61.6 \pm 16.8$ & $68.6 \pm 14.8$ \\
Platelet count $\left(\mathrm{x} 10^{9} / \mathrm{l}\right)$ & $30.7 \pm 67.8$ & $40.2 \pm 18.8$ & $50.1 \pm 85.6$
\end{tabular}

Results are presented as the mean \pm 2 standard deviations.

Table III. Changes in platelet count following treatment in the subgroups.

\begin{tabular}{lccr}
\hline Subgroup & n & $\begin{array}{c}\text { Count prior to treatment } \\
\left(\mathrm{x} 10^{9} / 1\right)\end{array}$ & $\begin{array}{c}\text { Count following } 3 \text { cycles } \\
\left(\mathrm{x} 10^{9} / \mathrm{l}\right)\end{array}$ \\
\hline Time from diagnosis to treatment $\leq 3$ months; IPSS $=0.5$ & 14 & $31.4 \pm 29.6$ & $58.6 \pm 25.8$ \\
Time from diagnosis to treatment $>3$ months; IPSS $=1.0$ & 16 & $26.5 \pm 48.6$ & $31.7 \pm 68.3$ \\
\hline
\end{tabular}

Results are presented as the mean \pm 2 standard deviations. IPSS, International Prognostic Scoring System.

with four cycles. Red blood cell transfusion was given to those with symptomatic anaemia, and platelet transfusions were given to those with platelet counts of $<6 \times 10^{9} / 1$ or those with obvious thrombocytopenic bleeding. None of the patients required EPO, cyclosporine (CSA) or iron chelation treatments.

Evaluation. Peripheral blood cell counts were monitored every 3-5 days, whereas liver and renal functions were reviewed every two weeks. The bone marrow morphology, cytogenetic analysis and FISH test were performed once the patients had undergone three cycles of treatment.

Statistical analysis. Statistical data were processed using SPSS statistical software version 13.0 (SPSS, Inc., Chicago, IL, USA), and comparisons among groups were conducted using a t-test. $\mathrm{P}<0.05$ was considered to indicate a statistically significant result.

\section{Results}

Changes in peripheral blood cell counts in the 30 patients following treatment. The efficacy of lenalidomide as a treatment for patients with intermediate-1 risk non-del (5q) MDS was demonstrated by the peripheral blood cell counts of the patients showing improvement following lenalidomide treatment. A total of 2-4 cycles of treatment resulted in the absolute neutrophil, haemoglobin and platelet counts of the patients increasing; however, the changes were not statistically significant $(\mathrm{P}>0.05)$. Analysis within the subgroups showed that the differences in the peripheral blood cell counts between the patients with scores of 0.5 and a duration of $\leq 3$ months between diagnosis and treatment and the patients with scores of 1.0 and a duration of $>3$ months between diagnosis and treatment were statistically significant $(\mathrm{P}<0.05)$. Platelet recovery in the former subgroup was more rapid and marked, with the fastest platelet recovery of $20 \mathrm{q}$ - observed in seven patients. However, patients with initial platelet counts of $<10 \times 10^{9} / 1$ and haemoglobin levels of $<45 \mathrm{~g} / \mathrm{l}$ demonstrated a poor response to the treatment (Tables II and III, respectively).

Evaluation of the bone marrow morphology and cytogenetic analysis. All patients became stable following three cycles of treatment; however, 17 cases with chromosomal abnormalities demonstrated no cytogenetic response, as confirmed by the FISH test.

Adverse events. In the first two weeks, $40 \%$ of the patients treated with lenalidomide exhibited mild rashes (12 cases); however, the majority of them recovered without any treatment. One case was treated with glucocorticoids and an HT-3 receptor antagonist. Within one week, 11 patients (36.67\%) who had initially shown abdominal distension experienced an improvement in the symptoms with continuous lenalidomide treatment. Following three cycles of treatment, one patient suffered from deep venous thrombosis (DVT) in the left lower limb, and although urokinase was administered, the thrombosis was not completely recanalised. In addition, all patients presented with liver and kidney dysfunctions.

\section{Discussion}

Lenalidomide exhibits immunological and biological properties, extending to cytokine modulation, T-cell costimulation and angiogenesis inhibition (6-8). Therefore, the effects of the drug on the MDS clone and the microenvironment may contribute to the therapeutic action. These effects include, but are not limited to, suppression of tumor necrosis factor- $\alpha$ and the induction of other inflammatory cytokines, such as interleukins-1 $\beta, 6,8$ and 12 ; costimulation of the T-cell-specific immune response; expansion of natural killer cells and enhancement of cytotoxicity; suppression of the endothelial response to angiogenic molecules; downregula- 
tion of cell-adhesion molecules; potentiation of EPO-receptor signaling and modification of lineage commitment. Furthermore, lenalidomide has been demonstrated to promote cell cycle arrest and display direct antineoplastic activity in a variety of cancer cell lines and primary specimens. Although a precise cellular target has not been identified to date, recent investigations have indicated that lenalidomide acts through the inhibition of phosphatase activity in the common deleted region (CDR) of 5q, which is important in cell cycle regulation through a defect in ribosomal protein function. As such, lenalidomide acts through direct cytotoxic mechanisms in patients with the del $(5 q)$ cytogenetic abnormality, in addition to affecting the bone marrow microenvironment in patients without this lesion (by suppressing the effects of proapoptotic and proinflammatory cytokines) (4,9-11).

The beneficial results of lenalidomide have been particularly evident for patients with del (5q) chromosome abnormalities $(10,12,13)$. A randomised clinical trial demonstrated that lenalidomide treatment may have been effective in improving the health-related quality of life (HRQL) outcomes of the tested patients (14). Lenalidomide has recently been approved by the FDA for the treatment of patients with low-risk MDS, particularly for patients with del (5q) (15). For patients without del $(5 q)$, however, the effects of lenalidomide remain unclear, and its potential use and activity in non-del (5q) MDS require further investigation $(16,17)$. A phase II study evaluated lenalidomide efficacy in 214 transfusion-dependent patients with low or intermediate-1 risk MDS without del (5q). The results showed that $26 \%$ of the non-del (5q) patients achieved transfusion independence following a median of 4.8 weeks of treatment (13). In a study by Sibon et al (18), 31 consecutive patients with lower-risk non-del (5q) MDS and anemia refractory to erythropoiesis-stimulating agents (ESA) were treated with lenalidomide in a compassionate programme. A total of 15 patients $(48 \%)$ demonstrated an erythroid response, including 10 of the 27 (37\%) previously transfusion-dependent (RBC-TD) patients who became transfusion-independent (RBC-TI). Out of these 15 patients, nine experienced a relapse, while the remaining six (40\%) were still responding and transfusion-free for at least 11(+)-31(+) months subsequently (median time, 24 months). There was a lower erythroid response rate in patients with refractory cytopaenia with multilineage dysplasia (27\% versus $60 \%)$; in addition, the response rate tended to be higher in patients treated with lenalidomide in combination with ESA (55\% versus $36 \%)$. The response duration was significantly longer in patients who became RBC-TI and in patients treated with lenalidomide following primary resistance to ESA. It was concluded that lenalidomide was an interesting second-line therapeutic option and that the combination of lenalidomide with ESAs in the context of the study required further investigation (18).

The present study aimed to observe the efficacy of lenalidomide in patients with intermediate-1 risk non-del (5q) MDS. It was observed that lenalidomide did not result in complete haematological remission in the patients, although it improved the peripheral blood cell counts in the patients with intermediate-1 risk MDS. Platelet recovery was more marked in the patients with IPSS scores of 0.5 and with a duration of $\leq 3$ months between diagnosis and treatment. The majority of patients achieved platelet transfusion independence, i.e. the safety level of non-spontaneous bleeding $\left(\geq 20 \times 10^{9} / 1\right)$. Furthermore, certain patients achieved red blood cell transfusion independence ( $\geq 60 \mathrm{~g} / \mathrm{l}$ ) following treatment. Patients with initial platelet and haemoglobin counts of $<10 \times 10^{9} / 1$ and $<45 \mathrm{~g} / \mathrm{l}$, respectively, demonstrated a poor response to the treatment. Patients with a duration of $<3$ months between diagnosis and treatment showed marked improvements in their blood cell counts, whereas those with a duration of $>3$ months between diagnosis and treatment demonstrated no apparent improvement. Therefore, lenalidomide is not a recommended treatment for patients with severe cytopaenia and with a prolonged duration between diagnosis and treatment.

At present, two phase III studies on lenalidomide are underway. One of these is known as the AZA-004 trial, which studies the effect of lenalidomide on the transformation of MDS into AML (19). The other study is examining the efficacy of lenalidomide in patients with non-del (5q) intermediate-1 risk MDS. It is expected that satisfactory results will be obtained from these two clinical trials.

The reported rate of DVT (VTE) for patients with MDS receiving lenalidomide during the first 2 years of post-marketing exposure has been revealed to be low $(0.53 \%)$. Disproportionality analysis demonstrated a statistically meaningful correlation between VTE and lenalidomide concomitantly used with ESAs in patients with MDS; however, the correlation was not statistically significant when lenalidomide was used in the absence of ESAs (20). Of the 30 patients examined in the current study, one patient suffered from DVT in the left lower limb following three cycles of treatment, and although urokinase was administered, the thrombosis was not completely recanalised. This result was not consistent with those presented in other studies, and more cases are required to make further observations.

In conclusion, lenalidomide has demonstrated efficacy in patients with intermediate-1 risk non-del (5q) MDS, and is indicated to be a safe form of treatment.

\section{References}

1. Haase D, Germing U, Schanz J, et al: New insights into the prognostic impact of the karyotype in MDS and correlation with subtypes: evidence from a core dataset of 2124 patients. Blood 110: 4385-4395, 2007.

2. Nimer SD: Myelodysplastic syndromes. Blood 111: 4841-4851, 2008.

3. Ebert BL, Galili N, Tamayo P, et al: An erythroid differentiation signature predicts response to lenalidomide in myelodysplastic syndrome. PLoS Med 5: e35, 2008.

4. Adès L, Boehrer S, Prebet T, et al: Efficacy and safety of lenalidomide in intermediate-2 or high-risk myelodysplastic syndromes with $5 q$ deletion: results of a phase 2 study. Blood 113: 3947-3952, 2009.

5. Greenberg PL, Attar E, Bennett JM, Bloomfield CD, De Castro CM, Deeg HJ, Foran JM, Gaensler K, Garcia-Manero G, Gore SD, Head D, Komrokji R, Maness LJ, Millenson M, Nimer SD, O'Donnell MR, Schroeder MA, Shami PJ, Stone RM, Thompson JE, Westervelt P; National Comprehensive Cancer Network: NCCN Clinical Practice Guidelines in Oncology: myelodysplastic syndromes. J Natl Compr Canc Netw 9: 30-56, 2011.

6. Haslett PA, Corral LG, Albert M and Kaplan G: Thalidomide costimulates primary human $\mathrm{T}$ lymphocytes, preferentially inducing proliferation, cytokine production, and cytotoxic responses in the CD8+ subset. J Exp Med 187: 1885-1892, 1998.

7. Davies FE, Raje N, Hideshima T, et al: Thalidomide and immunomodulatory derivatives augment natural killer cell cytotoxicity in multiple myeloma. Blood 98: 210-216, 2001. 
8. Chang DH, Liu N, Klimek V, et al: Enhancement of ligand-dependent activation of human natural killer $\mathrm{T}$ cells by lenalidomide: therapeutic implications. Blood 108: 618-621, 2006.

9. List A, Kurtin S, Roe DJ, et al: Efficacy of lenalidomide in myelodysplastic syndromes. N Engl J Med 352: 549-557, 2005.

10. List A, Dewald G, Bennett J, et al; Myelodysplastic Syndrome-003 Study Investigators: Lenalidomide in the myelodysplastic syndrome with chromosome 5q deletion. N Engl J Med 355: 1456-1465, 2006.

11. List AF: Lenalidomide - the phoenix rises. N Engl J Med 357 2183-2186, 2007.

12. Raza A, Reeves JA, Feldman EJ, et al: Phase 2 study of lenalidomide in transfusion-dependent, low-risk, and intermediate-1 risk myelodysplastic syndromes with karyotypes other than deletion 5q. Blood 111: 86-93, 2008.

13. Sekeres MA, Maciejewski JP, Giagounidis AA, et al: Relationship of treatment-related cytopenias and response to lenalidomide in patients with lower-risk myelodysplastic syndromes. J Clin Oncol 26: 5943-5949, 2008

14. Revicki DA, Brandenburg NA, Muus P, Yu R, Knight R and Fenaux P: Health-related quality of life outcomes of lenalidomide in transfusion-dependent patients with Lowor Intermediate-1-risk myelodysplastic syndromes with a chromosome $5 \mathrm{q}$ deletion: Results from a randomized clinical trial. Leuk Res 37: 259-265, 2013.
15. Komrokji RS and List AF: Role of lenalidomide in the treatment of myelodysplastic syndromes. Semi Oncol 38: 648-657, 2011.

16. Giagounidis AA: Lenalidomide for $\operatorname{del}(5 q)$ and non-del $(5 q)$ myelodysplastic syndromes. Semin Hematol 49: 312-322, 2012.

17. Sugimoto Y, Sekeres MA, Makishima H, et al: Cytogenetic and molecular predictors of response in patients with myeloid malignancies without del[5q] treated with lenalidomide. J Hematol Oncol 5: 4, 2012

18. Sibon D, Cannas G, Baracco F, et al; Groupe Francophone des Myélodysplasies: Lenalidomide in lower-risk myelodysplastic syndromes with karyotypes other than deletion $5 \mathrm{q}$ and refractory to ery thropoiesis-stimulating agents. Br J Haematol 156: 619-625, 2012.

19. Fenaux P, Giagounidis A, Selleslag DL, et al: RBC transfusion independence and safety profile of lenalidomide 5 or $10 \mathrm{mg}$ in Pts with low-or Int-1-risk MDS with Del5q: Results from a randomized phase III trial (MDS-004). Blood (ASH Annual Meeting abstracts) 114: 944, 2009.

20. Yang X, Brandenburg NA, Freeman J, et al: Venous thromboembolism in myelodysplastic syndrome patients receiving lenalidomide: results from postmarketing surveillance and data mining techniques. Clin Drug Investig 29:161-171, 2009. 